\title{
Asteroseismology of Very Low-Frequency Red Giants with Kepler: the Breakdown of the Asymptotic Relation
}

\author{
Douglas L. Compton ${ }^{1,2}$, Dennis Stello ${ }^{1,2}$, and Timothy R. Bedding ${ }^{1,2}$ \\ 1 Sydney Institute for Astronomy (SIfA), School of Physics, University of Sydney, Australia \\ 2 Stellar Astrophysics Centre, Department of Physics and Astronomy, Aarhus University, Denmark
}

\begin{abstract}
As the Kepler time series grew, so did the interest in the asteroseismology of very low-frequency solar-like oscillators. It is possible to accurately resolve modes for stars near the tip of the red giant branch. Our analysis of M-giant stars shows that both radial and non-radial modes are well defined in the spectra and form a triplet mode structure. By comparing the data to theoretical models we have successfully classified the angular degree and radial order of modes in 195 evolved red giants.
\end{abstract}

\section{Introduction}

We tracked the evolution of the adiabatic frequency pattern from the main sequence near to the tip of the red giant branch for a series of models [1]. The models were derived using the MESA 1M_pre_ms_to_wd test suite case [2], with the frequencies calculated using ADIPLS [3].

Our targets consisted of $195 \mathrm{M}$-giants pre-selected from the ground-based All Sky Automated Survey [4]. Long cadence Kepler data $(\Delta t \simeq 29.4 \mathrm{~min})$ spanning Q2-Q16 was used in the analysis. Before the power spectra were calculated, flux corrections were made to the time series. The main challenge arose from the fact that the period of the oscillations were often on the same time scale as the length of the quarters.

\section{Methodology}

Each star was categorised as either a 'fast' or 'slow' pulsator based on the number of intercepts between the first quarter (Q2) of data and its linear fit. We chose 15 crossings to be the lower bound for 'fast' pulsators, which corresponds to an oscillation period of approximately $1 \mu \mathrm{Hz}$. For 'fast' pulsators a linear fit was of the data was subtracted to each quarter before shifting the flux such that it equaled the average flux of the first quarter. This corrects for flux drifts due to the star moving relative to the aperture and jump discontinuities between quarters.

For 'slow' pulsators a linear fit was made to the five points on either side of the quarterly gaps before shifting the later quarter such that the two fits intersected at the center of the gap. However, if the gap between quarters was greater than 10 days the flux was adjusted to have an average flux equal to the first quarter.

Once the time series were stitched together the power spectra were calculated. We manually examined each spectrum to identify the radial order $n$ and angular degree $l$ of a prominent mode for each star, known as a reference mode with associated reference frequency $v_{\text {ref }}$. This frequency was matched to the grid of model by interpolating $v_{\text {ref }}$ between the frequencies of the corresponding modes in the adjacent models. This returned an associated model $v_{\max }$ and other stellar parameters through further interpolation of the models.

To obtain a more accurate result, 50 artificial spectra were created by interpolating the model grid $\pm 20 \%$ of the initial $v_{\max }$ value found in the previous step. Each mode was represented by a Lorentzian 
profile with width equal to the inverse of the observing time. The mode heights were attenuated from unity by a Gaussian envelope centered at $v_{\max }$ with full width half maximum equal to $0.25 v_{\max }$. The observed power spectrum was normalised by dividing the maximum power then correlated with the artificial spectra. This assigned theoretical stellar parameters based on the interpolated model with the highest correlation.

\section{Results}

The results are presented by plotting each spectrum as a horizontal band in grey scale according to their theoretical $v_{\max }$ shown in Fig. 1. The frequency axis of each spectrum was divided by the $v_{n=3, l=0}$ mode of the interpolated model. The dipole mode strongly deviates from the asymptotic relation [5] creating a triplet mode structure in the power spectra.

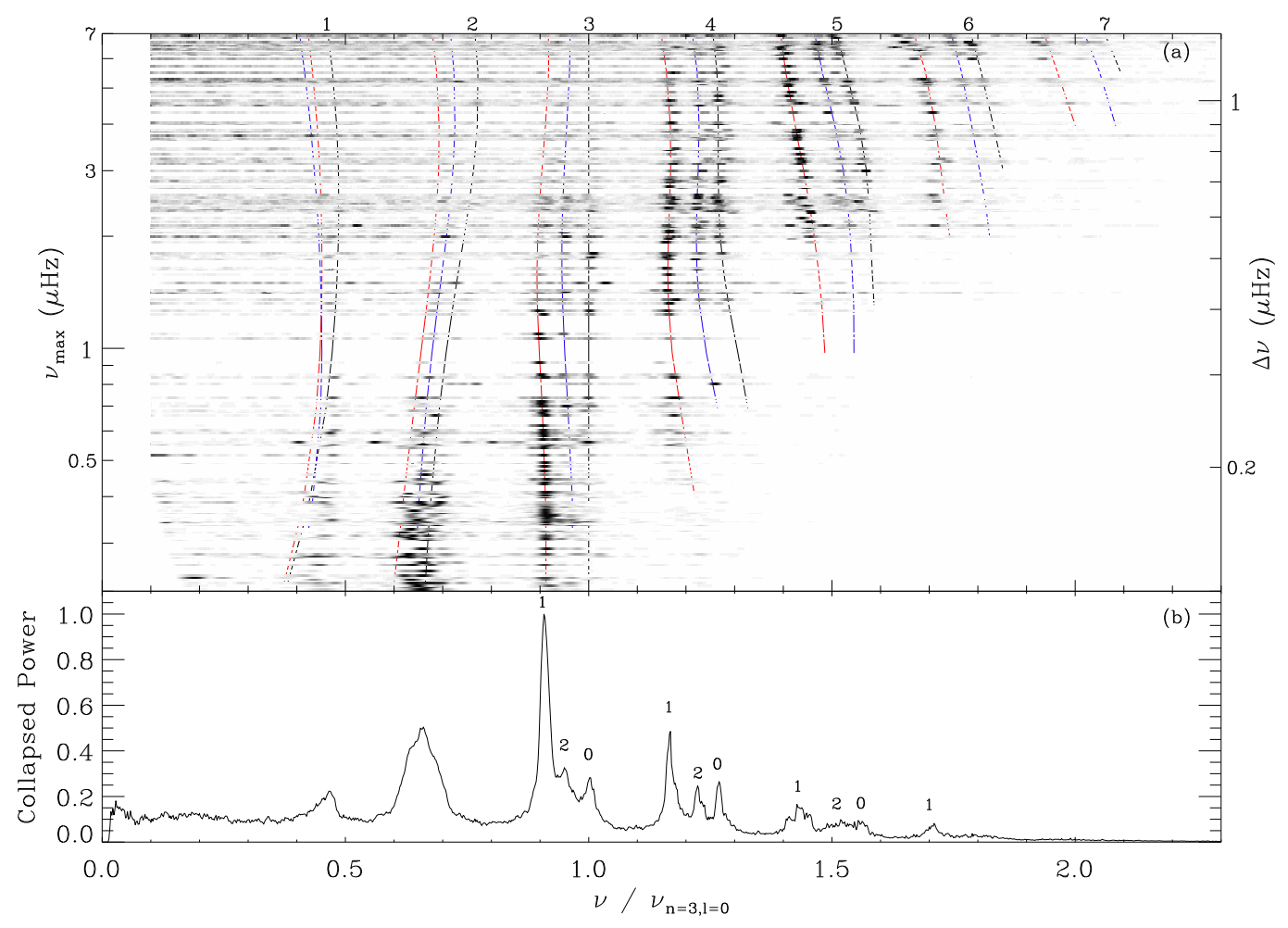

Fig. 1: (a) Observed power spectra of 195 Kepler stars. The models are plotted beneath the data with $l=0$ in black, $l=1$ in red, and $l=2$ in blue. The radial order is indicated above. (b) Vertically collapsed version of (a) with the numbers denoting the angular degree.

\section{References}

1. D. Stello, et al., ApJ, 788, L10 (2014)

2. B. Paxton, et al., ApJS, 208, 4 (2013)

3. J. Christensen-Dalsgaard, Ap\&SS, 316, 113 (2008)

4. A. Pigulski, et al., Acta Astron., 59, 33 (2009)

5. M. Tassoul, ApJS, 43, 469 (1980) 